Bulletin of the Section of Logic

Volume 45/2 (2016), pp. 125-140

http://dx.doi.org/10.18778/0138-0680.45.2.05

Andrzej Indrzejczak

\title{
SIMPLE DECISION PROCEDURE FOR S5 IN STANDARD CUT-FREE SEQUENT CALCULUS
}

\begin{abstract}
In the paper a decision procedure for $\mathbf{S 5}$ is presented which uses a cut-free sequent calculus with additional rules allowing a reduction to normal modal forms. It utilizes the fact that in S5 every formula is equivalent to some 1-degree formula, i.e. a modally-flat formula with modal functors having only boolean formulas in its scope. In contrast to many sequent calculi (SC) for $\mathbf{S 5}$ the presented system does not introduce any extra devices. Thus it is a standard version of SC but with some additional simple rewrite rules. The procedure combines the process of saturation of sequents with reduction of their elements to some normal modal form.
\end{abstract}

Keywords: Modal logic S5, decidability, normal forms, sequent calculus

\section{Introduction}

It is a well known fact that $\mathbf{S 5}$, despite numerous nice syntactic, semantic and algebraic properties, causes troubles in the framework of sequent calculus (SC). The first standard SC for S5, due to Ohnishi and Matsumoto $[21,22]$, was not cut-free, in contrast to SC devised for other popular modal logics like $\mathbf{T}$ or $\mathbf{S} 4$. In fact, they provided some solutions to this drawback (see section 4.) but the problem of finding fully satisfactory SC for S5 was in a sense open. Some proposals based on the standard form of SC were offered by Mints [19], Fitting [9], Takano [33] and Braüner [5]; they will be compared in the last section. There were also many nonstandard sequent calculi offering some solution to the problem. One can mention here for example: Kanger's indexed sequent calculus [14], Sato's approach [29], Belnap's display calculus [4] (see an exposition in Wansing [35]), Negri's labelled sequent calculus [20], Indrzejczak's double sequent calculus 
[13] or several approaches based on the idea of embedding sequents into sequents, like nested sequent calculus (Stouppa [31, 32] or Brünnler [6]) or tree-hypersequent calculus of Poggiolesi [25]. In particular, the problem was extensively examined on the ground of hypersequent calculi (HC), a generalised form of ordinary sequent calculi invented independently by Pottinger [26] and Avron [1]. They provided different solutions to the problem of cut-free formalization of $\mathbf{S 5}$ although in the same framework (Avron in [2]). In addition, other logicians, including Restall [28], Poggiolesi [23], Lahav [17], Kurokawa [16], Bednarska and Indrzejczak [3] provided different cut-free hypersequent calculi for $\mathbf{S 5}$.

On the other hand, different approaches to the problem of decision procedure for S5 were proposed on the ground of tableau methods (Kripke [15], Zeman [36], Fitting [9], Goré [11]), refutation metods (Skura [30]), resolution (Farinas del Cerro and Herzig [8]) or application of normal forms. Tableau-based approaches are quite close to approaches based on (cut-free) $\mathrm{SC}$ and may be essentially treated as belonging to the same group. The remaining solutions are often based on the fact that in $\mathbf{S 5}$ every formula is equivalent to some 1-degree formula, i.e. a modally-flat formula with modal functors having only boolean formulas in its scope. The latter approach is usually based on the application of some preprocessing step transforming any formula into some normal form. It was first proposed by Carnap [7] (also Wajsberg [34]); an exposition of such a procedure may be found in Hughes and Cresswell [12].

In what follows we provide an SC which is an extension of Ohnishi and Matsumoto system for S5 with some additional simple rules. A decision procedure which is defined on this basis utilizes the fact that the (additional) rules of the system allows us to reduce every formula to its 1-degree counterpart. However, there is no preprocessing step of transformation a formula into some normal form. Instead, the whole procedure, and a completeness proof induced by it, is in the spirit of SC and tableau (downward) saturation procedures, with additional transformation steps involved.

\section{S5 - basic facts}

Let us recall the basic facts concerning modal logic $\mathbf{S 5}$ in standard characterization, i.e., as an axiomatic system adequate with respect to suitable classes of relational (Kripke) frames. We will use standard monomodal language with countable set $P V$ of propositional variables, $\square$ - unary modal 
necessity operator, and ordinary boolean constants. One can axiomatize S5 by adding to some system for classical propositional logic the following schemata:

$$
\begin{aligned}
& \mathrm{K} \square(\varphi \rightarrow \psi) \rightarrow(\square \varphi \rightarrow \square \psi) \\
& \mathrm{T} \square \varphi \rightarrow \varphi \\
& 4 \square \varphi \rightarrow \square \square \varphi \\
& 5 \neg \square \varphi \rightarrow \square \neg \square \varphi
\end{aligned}
$$

Instead of 5 one can use:

$$
\mathrm{B} \neg \varphi \rightarrow \square \neg \square \varphi
$$

The system is closed under MP (modus ponens) and the rule of necessitation (of every thesis).

The simplest semantical characterization of S5 may be obtained by means of Kripke frames with no accessibility relation. A model for S5 is thus any pair $\mathfrak{M}=\langle\mathcal{W}, V\rangle, \mathcal{W} \neq \emptyset$ and $V: P V \longrightarrow \mathcal{P}(\mathcal{W})$. Satisfaction in a world of a model is inductively defined in the following way:

- $\mathfrak{M}, w \models \varphi$ iff $w \in V(\varphi)$, for any $\varphi \in P V$

- $\mathfrak{M}, w \models \neg \varphi$ iff $\mathfrak{M}, w \not \models \varphi$

- $\mathfrak{M}, w \models \varphi \wedge \psi$ iff $\mathfrak{M}, w \models \varphi$ and $\mathfrak{M}, w \models \psi$

- $\mathfrak{M}, w \models \varphi \vee \psi$ iff $\mathfrak{M}, w \models \varphi$ or $\mathfrak{M}, w \models \psi$

- $\mathfrak{M}, w \models \varphi \rightarrow \psi$ iff $\mathfrak{M}, w \not \models \varphi$ or $\mathfrak{M}, w \models \psi$

- $\mathfrak{M}, w \models \square \varphi$ iff $\mathfrak{M}, v \models \varphi$ for any $v \in \mathcal{W}_{\mathfrak{M}}$

A formula is $\mathbf{S 5}$-valid iff it is true in every world of every model.

Although cut is not eliminable from ordinary SC for S5 (see section 4.) we can enrich the set of rules in such a way that we can dispense with cut and prove completeness of this enriched system (without cut). Moreover, we obtain a simple decision procedure for S5. The idea is based on the fact that every modal formula in $\mathbf{S 5}$ is equivalent to modal formula of 1-degree. In such a formula no $\square$ is in the scope of another $\square$. This was first examined by Wajsberg [34] and Carnap [7]. One can find an exposition of such a decision procedure running by reduction to modal normal form in Hughes and Cresswell [12]. Here we will apply SC resources and instead of preliminary transformation of any formula to its modal equivalent of 1-degree we will obtain rather a class of sequents in a special form but the key step of reduction to 1-degree modal formula is similar. 


\section{The calculus}

We will provide a calculus which is based on the formalization of S5 due to Ohnishi and Matsumoto but contains also a set of additional rules which will be stated with the help of the following conventions:

- $\varphi$ is an M-formula if it is of the form $\square \psi$ or $\neg \square \psi$.

- $\square \Gamma=\{\square \varphi: \varphi \in \Gamma\}$

- $\square[\varphi]$ means that there is $\square \psi$ such that $\varphi$ is a subformula (not necessarily proper) of $\psi$.

- $S, \varphi$ means that $\varphi$ is either in the antecedent or in the succedent of a sequent $S$.

Sequents are built from finite sets of formulas. Axioms are of the form $\Gamma \Rightarrow \Delta$ with $\Gamma \cap \Delta$ nonempty. There are no structural rules, only the following logical rules:

$$
\begin{aligned}
& (\neg) \frac{\Gamma \Rightarrow \Delta, \varphi}{\neg \varphi, \Gamma \Rightarrow \Delta} \\
& (\Rightarrow \neg) \frac{\varphi, \Gamma \Rightarrow \Delta}{\Gamma \Rightarrow \Delta, \neg \varphi} \\
& (\wedge \Rightarrow) \frac{\varphi, \psi, \Gamma \Rightarrow \Delta}{\varphi \wedge \psi, \Gamma \Rightarrow \Delta} \\
& (\Rightarrow \wedge) \frac{\Gamma \Rightarrow \Delta, \varphi \quad \Gamma \Rightarrow \Delta, \psi}{\Gamma \Rightarrow \Delta, \varphi \wedge \psi} \\
& (\Rightarrow \vee) \quad \frac{\Gamma \Rightarrow \Delta, \varphi, \psi}{\Gamma \Rightarrow \Delta, \varphi \vee \psi} \\
& (\vee \Rightarrow) \frac{\varphi, \Gamma \Rightarrow \Delta \quad \psi, \Gamma \Rightarrow \Delta}{\varphi \vee \psi, \Gamma \Rightarrow \Delta} \\
& (\Rightarrow \rightarrow) \frac{\varphi, \Gamma \Rightarrow \Delta, \psi}{\Gamma \Rightarrow \Delta, \varphi \rightarrow \psi} \\
& (\rightarrow \Rightarrow) \frac{\Gamma \Rightarrow \Delta, \varphi \quad \psi, \Gamma \Rightarrow \Delta}{\varphi \rightarrow \psi, \Gamma \Rightarrow \Delta} \\
& (\square \Rightarrow) \frac{\square \varphi, \varphi, \Gamma \Rightarrow \Delta}{\square \varphi, \Gamma \Rightarrow \Delta} \\
& (\Rightarrow \square) \frac{\square \Gamma \Rightarrow \square \Delta, \varphi}{\Pi, \square \Gamma \Rightarrow \square \Delta, \Sigma, \square \varphi}
\end{aligned}
$$

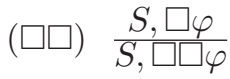

$$
\begin{aligned}
& (\square \neg \square) \\
& \frac{S, \neg \square \varphi}{S, \square \neg \square \varphi} \\
& (\square \neg \neg) \frac{S, \square[\varphi]}{S, \square[\neg \neg \varphi]} \\
& (\square \wedge) \frac{S, \square \varphi \wedge \square \psi}{S, \square(\varphi \wedge \psi)} \\
& (\square \vee)^{*} \frac{S, \square \varphi \vee \square \psi}{S, \square(\varphi \vee \psi)} \\
& (\square \rightarrow) \frac{S, \square[\neg \varphi \vee \psi]}{S, \square[\varphi \rightarrow \psi]} \\
& (\square \vee \wedge) \frac{S, \square[(\varphi \vee \psi) \wedge(\varphi \vee \chi)]}{S, \square[\varphi \vee(\psi \wedge \chi)]} \\
& (\square \neg \wedge) \quad \frac{S, \square[\neg \varphi \vee \neg \psi]}{S, \square[\neg(\varphi \wedge \psi)]} \\
& (\square \neg \vee) \frac{S, \square[\neg \varphi \wedge \neg \psi]}{S, \square[\neg(\varphi \vee \psi)]}
\end{aligned}
$$

* At least one of the disjuncts is M-formula. 
The last nine rules on the list will be called rewrite rules. Since their schemata are expressed by means of the last convention we have in fact two rules in each case: one for a transformation of a displayed formula in the antecedent, and the other for the same transformation but in the succedent. Note also that the last four and ( $\square \neg \neg$ ) allow for making transformations on subformulas of modal formulas. All of them serve for retaining a necessary minimum of a procedure of reduction to normal form. A side condition for $(\square \vee)$ follows from the restricted distributivity of $\square$ and $\vee$.

Two remarks concerning the selection and the shape of our rules may be of interest. Note that the calculus is redundant. One may easily notice that the effect of the application of $(\square \square)$ and $(\square \neg \square)$ in the antecedent is covered by $(\square \Rightarrow)$, however these special applications need no repetition of principal formula in the premiss, so it is simpler to formulate them as general rules of elimination for duplicated modalities.

A proof is defined in a standard way as a binary tree of sequents. A proof-tree is defined similarly but we admit that some leaves are not axiomatic.

Notice that in our system we obtain a simple cut-free proof of B:

$$
\begin{gathered}
(\square \Rightarrow) \frac{p, \square p \Rightarrow p}{\square p \Rightarrow p} \\
(\neg \Rightarrow) \frac{\square p, \neg p \Rightarrow}{(\Rightarrow \neg) \frac{\neg p \Rightarrow \neg \square p}{\square}} \\
(\square \neg \square) \frac{\neg p \Rightarrow \square \neg \square p}{\Rightarrow \neg p \rightarrow \square \neg \square p}
\end{gathered}
$$

Still we cannot prove cut elimination theorem constructively for such a system but only define a suitable proof-search procedure which shows that it is an adequate cut-free system for $\mathbf{S 5}$.

A sequent $S$ is reducible to a set of sequents $S_{1}, \ldots S_{n}, n \geq 1$ iff there exists a proof-tree with $S$ as a root and $S_{1}, \ldots, S_{n}$ as all leaves.

We may translate sequents in a standard way into formulas of $\mathbf{S 5}$ by treating antecedents as conjunctions, succedents as disjunctions and $\Rightarrow$ as $\rightarrow$. A rule is validity-preserving (in S5) iff under translation the conclusion is valid whenever all premises are valid. A rule is invertible iff validity of translated conclusion implies validity of translation of each premise.

Theorem 1 (Soundness). If $\vdash \Gamma \Rightarrow \Delta$, then $\models \wedge \Gamma \rightarrow \vee \Delta$

PRoOF: follows from the fact that all rules are validity-preserving. 
Lemma 1 (Invertibility). All rules except $(\Rightarrow \square)$ are invertible.

Note that we could delete in the schema of $(\Rightarrow \square)$ sets $\Pi, \Sigma$ in the conclusion and obtain invertible version of this rule but then we need explicit rules of weakening which are not invertible either.

In order to state our procedure we need to introduce more terminology and prove some preliminary results.

We will say that $\varphi$ is 0 -formula if it is boolean, i.e. does not contain $\square$. If it is an M-formula $\square \psi$ or $\neg \square \psi$, then it is of the 1-degree, or simply 1 -formula if $\psi$ is 0 -formula. It is of the $n$th degree $(n>1)$, or simply $n$-formula if its every subformula which is M-formula is of the degree at most $n-1$.

M-formula is M-normal formula if it is 1-formula or if it is $n$-formula of the form $\square\left(\psi_{1} \wedge \cdots \wedge \psi_{k}\right), k \geq 1$, where each $\psi_{i}$ is either M-formula (of at most $n-1$ degree) or M-clause. The latter are of the form $\psi_{1} \vee \cdots \vee \psi_{i}, i \geq 1$ where each disjunct is a literal or M-formula (of at most $n-1$ degree).

Note that we do not require that in 1-formulas its boolean subformula must be in conjunctive normal form. Moreover, we freely use associativity and commutativity hence in practice we generalize the notion of conjunction and disjunction to arbitrary finite number of elements including one.

$S$ is an M-sequent iff it contains at least one M-formula. M-sequent is $n$-sequent $(n \geq 1)$ if each M-formula in it is of at most degree $n$; in particular, 1-sequent is a sequent with all M-formulas of degree 1. Any M-sequent will be called reduced iff in addition to M-formulas it contains only atomic formulas (if any). Finally by an atomic sequent we mean a sequent of the form $\Gamma \Rightarrow \Delta$ with $\Gamma \cup \Delta$ containing only atomic formulas.

Lemma 2. Each $S, \square \psi$ is reducible to $S, \square \psi^{\prime}$ with $\square \psi^{\prime}$ being M-normal formula of the same degree as $\square \psi$.

Proof: The proof goes like in classical case by repeated application of rewrite rules to $\psi$.

LEMMA 3. Each $n$-sequent $(n>1)$ is reducible to a set of $n-1$-sequents.

Proof: Consider arbitrary $n$-formula and reduce it first to M-normal form by lemma 2 . Thus we have a sequent $S, \square \psi$ with some $\square \psi$ being M-normal. We will show by induction on the length of $\psi$ that it is reducible. There are three cases. 
(a) $\psi$ is a $k$-element conjunction $(k>1)$ of the form $\chi_{1} \wedge \cdots \wedge \chi_{k}$. Apply $(\square \wedge)$ to get $\square \chi_{1} \wedge \square\left(\chi_{2} \wedge \cdots \wedge \chi_{k}\right)$ and then either $(\wedge \Rightarrow)$ or $(\Rightarrow \wedge)$ depending on the position of this formula in a sequent. This way we obtain either a one branch proof-tree with a leaf $S, \square \chi_{1}, \ldots, \square\left(\chi_{2} \wedge \cdots \wedge \chi_{k}\right)$ (with both modal formulas in the antecedent) or a proof-tree with two leaves for each of these two formulas in the succedent of $S$. By the induction hypothesis each of $\square \chi_{1}$ and $\square\left(\chi_{2} \wedge \cdots \wedge \chi_{k}\right)$ is reducible to $n-1$ degree.

(b) $\psi$ is an $i$-element M-clause $(i>1)$ of the form $\chi_{1} \vee \cdots \vee \chi_{i}$ and there must be at least one disjunct which is M-formula, let it be $\chi_{1}$. We apply $(\square \vee)$ to get $\square \chi_{1} \vee \square\left(\chi_{2} \vee \cdots \vee \chi_{i}\right)$ and then either $(\vee \Rightarrow)$ or $(\Rightarrow \vee)$ depending on the position of $\square \psi$ in a sequent. In a similar way as in the preceding case we obtain a proof-tree where each of $\square \chi_{1}$ and $\square\left(\chi_{2} \vee \cdots \vee \chi_{i}\right)$ is by the induction hypothesis reducible to $n-1$ degree.

(c) $\psi$ is M-formula which is directly reducible to degree $n-1$ by the application of $(\square \square)$ or $(\square \neg \square)$ with $(\neg \Rightarrow)$ or $(\Rightarrow \neg)$. Incidentally this is the base step of our induction.

The procedure is repeated with respect to remaining M-formulas of degree $n$ (if any) until all leaves contain only M-formulas of at most $n-1$ degree.

As a corollary we obtain:

LEMMA 4. Each $n$-sequent $(n>1)$ is reducible to a set of 1 -sequents.

Now we will sketch a decision procedure in an indeterministic manner. In particular we do not specify if we build a proof-tree in a depth-first or breadth-first manner, or do not establish the order of operations. It goes in stages:

1. Start with $S$ and, if it contains at least one compound 0-formula apply only boolean rules, otherwise go to stage 2 . Continue with boolean rules until each nonaxiomatic leaf is an atomic sequent or reduced M-sequent of the form $\Gamma, \square \Pi \Rightarrow \square \Sigma, \Delta$, where $\Gamma$ and $\Delta$ are empty or atomic and $\Pi \cup \Sigma$ is nonempty. There are three possible outcomes: (a) all leaves are axiomatic$\vdash S$; (b) at least one leaf is nonaxiomatic and atomic - we define one-world model falsifying $S$ (c) all nonaxiomatic leaves are reduced M-sequents - we go to stage 2 , or - if all such leaves are already 1-modal - to stage 3 .

2. To each reduced M-sequent apply lemmata 2-4 until either you get axiomatic sequents in all leaves or at least one nonaxiomatic leaf with reduced 1-sequent, i.e. of the form $\Gamma, \square \Pi \Rightarrow \square \Sigma, \Delta$, where $\Gamma$ and $\Delta$ are 
empty or atomic and nonempty $\Pi \cup \Sigma$ contain only 0 -formulas. Note that in this stage there is no possibility of reduction to atomic nonaxiomatic leaves; $\Gamma$ and $\Delta$ are fixed and the set of M-formulas may only increase.

3. To each 1-modal sequent $\Gamma, \square \Pi \Rightarrow \square \Sigma, \Delta$ apply:

3.0. If $\square \Pi$ is nonempty apply $(\square \Rightarrow)$ to all elements of $\square \Pi$ obtaining $\Gamma, \Pi, \square \Pi \Rightarrow \square \Sigma, \Delta$ and then repeat stage 1 . If all leaves are axiomatic or $\square \Sigma$ is empty then we stop with a proof or one-world falsifying model. Otherwise $\square \Sigma=\left\{\square \varphi_{1}, \ldots, \square \varphi_{n}\right\}$ and we continue with:

3.i. (for $i=1,2, \ldots, n)$ apply successively $(\Rightarrow \square)$ to $\square \varphi_{i}$, then $(\square \Rightarrow)$ to all elements of $\square \Pi$ and then repeat stage 1. Again we stop if all leaves are axiomatic or continue with $\square \varphi_{i+1}$.

Each time in every nonaxiomatic leaf the number of boxed formulae in the succedent is diminished by 1 . This way after the last round (i.e. applied to $\left.\square \varphi_{n}\right)$, if we got some nonaxiomatic leaves they have only atoms on both sides and $\square \Pi$ in the antecedent. Note that in every substage $3 . i(\square \Rightarrow)$ is applied to all elements of $\square \Pi$ and, since elements of $\Pi$ are 0 -formulas, by repetition of stage 1 we finally obtain all atoms generated from $\Pi$. Hence we can treat all such leaves as atomic even if $\square \Pi$ is nonempty.

Two examples may help to grasp the idea of how the procedure works. First, an example of a proof:

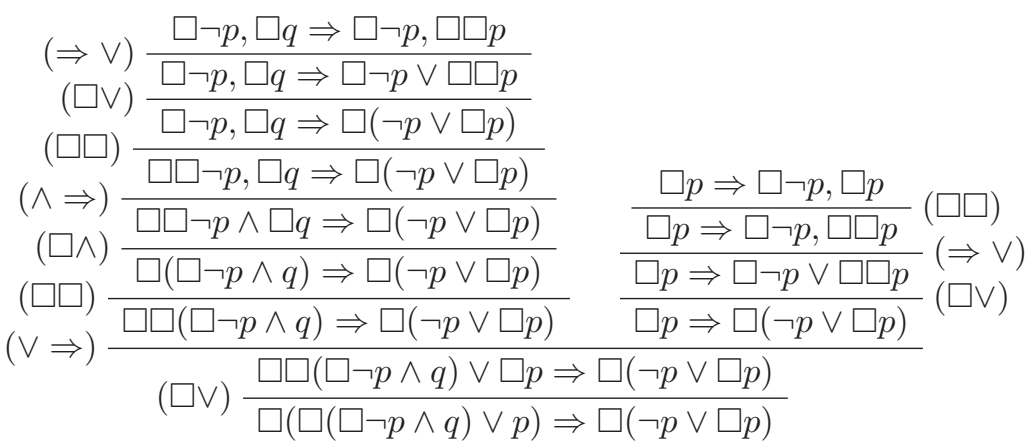

Since a root-sequent is a reduced M-sequent we start with performance of stage 2. Also stage 3 is not needed since both branches finish with axioms already at stage 2 .

In the second example we demonstrate a disproof realised in a depthfirst manner, always exploring the leftmost branch first. Since a root- 
sequent is already reduced M-sequent we start with stage 2 and finish with axiomatic sequent on the leftmost leaf.

$$
\begin{aligned}
& (\neg \Rightarrow) \frac{\square(p \rightarrow q) \Rightarrow \square q, \square(p \rightarrow q)}{\neg \square(p \rightarrow q), \square(p \rightarrow q) \Rightarrow \square q} \\
& (\vee \Rightarrow) \frac{\square \neg \square(p \rightarrow q), \square(p \rightarrow q) \Rightarrow \square q}{\square \neg \square(p \rightarrow q) \vee \square \neg \square \neg q, \square(p \rightarrow q) \Rightarrow \square q} \\
& (\Rightarrow \neg) \frac{\square \square(p \rightarrow q) \vee \square \rightarrow \neg q, \square(p \rightarrow q) \Rightarrow \square q}{\square \neg \square(p \rightarrow q) \vee \square \neg \square \neg q \Rightarrow \neg \square(p \rightarrow q), \square q}
\end{aligned}
$$

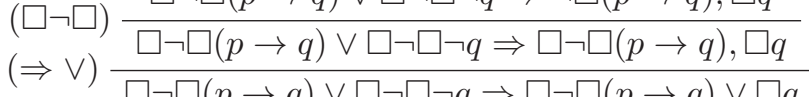

$$
\begin{aligned}
& (\square \vee) \frac{\square \neg \square(p \rightarrow q) \vee \square \neg \square \neg q \Rightarrow \square \neg \square(p \rightarrow q) \vee \square q}{\square \neg \square(p \rightarrow q) \vee \square \neg \square \neg q \Rightarrow \square(\neg \square(p \rightarrow q) \vee q)} \\
& (\square \rightarrow) \frac{\square}{\square \neg \square(p \rightarrow q) \vee \square \neg \square \neg q \Rightarrow \square(\square(p \rightarrow q) \rightarrow q)} \\
& (\square \vee) \frac{\square(\neg \square(p \rightarrow q) \vee \neg \square \neg q) \Rightarrow \square(\square(p \rightarrow q) \rightarrow q)}{\square(\square) \frac{\square(p \rightarrow q) \rightarrow \neg \square \neg q) \Rightarrow \square(\square(p \rightarrow q) \rightarrow q)}{\square(\square(p)}}
\end{aligned}
$$

Now we continue with the right branch and obtain the following tree:

$$
\begin{aligned}
& \frac{\square(p \rightarrow q), q \Rightarrow p \quad \square(p \rightarrow q), q \Rightarrow}{(\Rightarrow \neg) \frac{\square(p \rightarrow q), p \rightarrow q, q \Rightarrow}{\square(p \rightarrow q), p \rightarrow q \Rightarrow \neg q}}(\rightarrow \Rightarrow) \\
& (\square \Rightarrow) \frac{\square(p \rightarrow q), p \rightarrow q \Rightarrow \neg q}{\square(p \rightarrow q) \Rightarrow \neg q} \\
& (\Rightarrow \square) \frac{\square(p \rightarrow q) \Rightarrow \neg q}{\square(p \rightarrow q) \Rightarrow \square} \\
& (\rightarrow \Rightarrow) \frac{\square(p \rightarrow q) \Rightarrow \square \neg q, q, p}{\square(p \rightarrow q), p \rightarrow q \Rightarrow \square \neg q, q} \\
& \begin{array}{c}
(\Rightarrow \Rightarrow) \\
(\Rightarrow \square) \frac{\square(p \rightarrow q) \Rightarrow \square \neg q, q}{\square(p \rightarrow q) \Rightarrow \square \neg q, \square q, p}
\end{array} \\
& (\rightarrow \Rightarrow) \frac{\square(p \rightarrow q) \Rightarrow \square \neg q, \square q, p}{\square(p \rightarrow q), p \rightarrow q} \\
& (\square \Rightarrow) \frac{\square(p \rightarrow q), p \rightarrow q \Rightarrow \square \neg q, \square q}{\square(p \rightarrow q) \Rightarrow \square \neg q, \square q} \\
& (\neg \Rightarrow) \frac{\square(p \rightarrow q) \Rightarrow \square \neg q, \square q}{\square(p \rightarrow q), \neg \square \neg q \Rightarrow \square q} \\
& (\square \neg \square) \frac{\square(p \rightarrow q), \neg \square \neg q \Rightarrow \square q}{\square(p \rightarrow q), \square \neg \square \neg q \Rightarrow \square q}
\end{aligned}
$$

where $S_{1}$ denotes $q, \square(p \rightarrow q) \Rightarrow \square \neg q, q$ and $S_{2}$ denotes $q, \square(p \rightarrow q) \Rightarrow$ $\square \neg q, \square q$. A tree shows how after performing stage 2 we realize also stages 3.0 (below the first branching), 3.1 (above the first branching) and 3.2 (above the second branching) interleaved with repetitions of stage 1. Both leaves on the left are not axiomatic and the only boxed formula in their 
antecedents was already dealt with by means of $(\square \Rightarrow)$ in this substage, hence we can stop and build a 3-world falsifying model for a root-sequent.

The procedure yields decidability of $\mathbf{S 5}$ and completeness of this SC. The former is a consequence of subformula-property of all rules.

Let us sketch a completeness proof. We assume that $\nvdash \Gamma \Rightarrow \Delta$ and show that there is a model falsifying this sequent.

Let us consider a completed tree for $\Gamma \Rightarrow \Delta$ with at least one open branch $\mathcal{B}$. We assume that all stages were realised in the process of construction of this branch. Otherwise, (if it finished with nonaxiomatic atomic sequent in stage 1) we are done classically - with one-world model. We divide this branch into $n+1$ segments. The first segment starts with reduced 1-sequent $\Gamma^{\prime}, \square \Pi \Rightarrow \Delta^{\prime}, \square \varphi_{1}, \ldots, \square \varphi_{n}$ obtained as an input of stage 2 and ends with the first application of $(\Rightarrow \square$ ) (counting upwards). Thus the last element of this segment is also a reduced 1-sequent $\Gamma^{\prime \prime}, \square \Pi \Rightarrow \Delta^{\prime \prime}, \square \varphi_{1}, \ldots, \square \varphi_{n}$ being an input of stage 3.0 . (it is possible that there are no modal formulas in the succedent and we finish in stage 3.0 - again one-world model is enough). The next segment starts with $\square \Pi \Rightarrow \varphi_{1}, \square \varphi_{2}, \ldots, \square \varphi_{n}$ and ends with the second application of $(\Rightarrow \square$ ). Similarly for the other segments with the last one ending with nonaxiomatic leaf having no modal formulas in the succedent.

This way we divide our open branch into segments corresponding to succesive applications of $(\Rightarrow \square)$. For each segment $i$ we define an ordered pair $\left(\Gamma_{i}, \Delta_{i}\right)$ such that $\Gamma_{i}$ and $\Delta_{i}$ are unions of all formulas occuring respectively in the antecedents, and in the succedents of all sequents from segment $i$. One may easily check that each $\left(\Gamma_{i}, \Delta_{i}\right)$ is consistent, i.e. $\Gamma_{i} \cap \Delta_{i}=\emptyset$, and downward saturated in the following sense:

1. if $\neg \varphi \in \Gamma_{i}$, then $\varphi \in \Delta_{i}$

2. if $\neg \varphi \in \Delta_{i}$, then $\varphi \in \Gamma_{i}$

3. if $\varphi \wedge \psi \in \Gamma_{i}$, then $\varphi \in \Gamma_{i}$ and $\psi \in \Gamma_{i}$

4. if $\varphi \wedge \psi \in \Delta_{i}$, then $\varphi \in \Delta_{i}$ or $\psi \in \Delta_{i}$

5. if $\varphi \vee \psi \in \Gamma_{i}$, then $\varphi \in \Gamma_{i}$ or $\psi \in \Gamma_{i}$

6. if $\varphi \vee \psi \in \Delta_{i}$, then $\varphi \in \Delta_{i}$ and $\psi \in \Delta_{i}$

7. if $\varphi \rightarrow \psi \in \Gamma_{i}$, then $\varphi \in \Delta_{i}$ or $\psi \in \Gamma_{i}$

8. if $\varphi \rightarrow \psi \in \Delta_{i}$, then $\varphi \in \Gamma_{i}$ and $\psi \in \Delta_{i}$

This is secured by successive realizations of stage 1 inside each segment. 
Now we can define a falsifying model $\mathfrak{M}_{\mathcal{B}}$ containing $n+1$ worlds corresponding to segments of $\mathcal{B}$. Thus the first segment ending with the lowest application of $(\Rightarrow \square)$ corresponds to $w_{0}$, whereas each segment between $i$ th and $i+1$ application of $(\Rightarrow \square)$ corresponds to the world $w_{i}$ of a falsifying model. Valuation is defined for each atom $p: V(p)=\left\{w_{i}: p \in \Gamma_{i}\right\}$.

One may easily prove:

Lemma 5 (Truth-lemma). For each $\left(\Gamma_{i}, \Delta_{i}\right)$ :

- if $\varphi \in \Gamma_{i}$, then $\mathfrak{M}_{\mathcal{B}}, w_{i} \vDash \varphi$;

- if $\varphi \in \Delta_{i}$, then $\mathfrak{M}_{\mathcal{B}}, w_{i}, \not \neq \varphi$.

For boolean formulas it follows from downward saturation. For 1-modal formula $\square \psi$ in $\Gamma_{i}$ we must show that $\psi$ is in every $\Gamma_{k}, 0 \leq k \leq n$ and this is guaranteed by the first phase of stages $3.0-3 . n$. For 1 -modal formula $\square \varphi$ in $\Delta_{i}$ we must show that there is some $\Delta_{k}$ such that $\varphi \in \Delta_{k}$. This is also secured by construction of the procedure since each $\square \varphi_{i}$ has a segment $i$ with $\varphi_{i} \in \Delta_{i}$. Hence $\mathfrak{M}_{\mathcal{B}}$ is $\mathbf{S} 5$-model falsifying $\Gamma^{\prime}, \square \Pi \Rightarrow \Delta^{\prime}, \square \varphi_{1}, \ldots, \square \varphi_{n}$ in $w_{0}$ since $\Gamma^{\prime} \cup \square \Pi \subseteq \Gamma_{0}$ and $\Delta^{\prime} \cup\left\{\square \varphi_{1}, \ldots, \square \varphi_{n}\right\} \subseteq \Delta_{0}$. Consequently, also $\Gamma \Rightarrow \Delta$ is falsified by $w_{0}$ since all rules applied in stage 1 and 2 were invertible. Thus we obtain:

Theorem 2 (Completeness). If $\models \Gamma \Rightarrow \Delta$, then $\vdash \Gamma \Rightarrow \Delta$

\section{Comparison}

It may be instructive to compare our solution with some other cut-free SC for S5 based on ordinary Gentzen calculus. They may be roughly divided into such systems for which cut elimination (of some sort) was proved but which are not necessarily good for proof search, and such systems which yield a decision procedure but it is difficult to prove for them cut elimination constructively.

Ohnishi and Matsumoto provided the first SC for S5. They have used as a basis Gentzen's LK which operates on sequents built from finite lists of formulas so they have used additionaly structural rules of weakening, contraction and permutation. For $\square$ they introduced the following rules:

$$
(\square \Rightarrow) \frac{\varphi, \Gamma \Rightarrow \Delta}{\square \varphi, \Gamma \Rightarrow \Delta} \quad(\Rightarrow \square) \quad \begin{aligned}
& \square \Gamma \Rightarrow \square \Delta, \varphi \\
& \square \Gamma \Rightarrow \square \Delta, \square \varphi
\end{aligned}
$$

instead of our contraction- and weakening-absorbing rules. The following proof of B shows that cut is indispensable in this formalization of $\mathbf{S 5}$. 


$$
\begin{gathered}
(\Rightarrow \neg) \frac{\square p \Rightarrow \square p}{\Rightarrow \neg \square p, \square p} \quad \frac{p \Rightarrow p}{\square p \Rightarrow p} \\
\left.(\Rightarrow \square) \frac{\square}{\Rightarrow \square \neg \square p, \square p} \quad \frac{\square p}{(C u t)}\right) \\
(\neg \Rightarrow) \frac{\Rightarrow \square \neg \square p, p}{\neg p \Rightarrow \square \neg \square p} \\
(\Rightarrow \rightarrow) \frac{\Rightarrow \neg p \rightarrow \square \neg \square p}{\Rightarrow}
\end{gathered}
$$

Actually there are two solutions in Ohnishi and Matsumoto [21, 22] to the problem. One is based on the same idea of using only modal 1-formulas. There is no procedure for reducing S5 M-formulas to their equivalents of 1-degree but it is shown that cut is eliminable in the calculus restricted to 1-formulas. The second solution is by translation based on the following:

THEOREM 3. $\vdash_{S 5} \varphi$ iff $\vdash_{S 4} \square \diamond \square \varphi$

Since $\mathbf{S} 4$ has cut-free SC we obtain indirectly a decision procedure for S5. Note that in fact it may be simplified since we know that in $\mathbf{S} 4 \vdash \square \varphi$ iff $\vdash \varphi$. So in order to provide a proof for $\varphi$ in $\mathbf{S} 5$ it is enough to find a cut-free proof of $\square \neg \square \varphi \Rightarrow$ in SC for $\mathbf{S} 4$. Here is a cut-free proof of B:

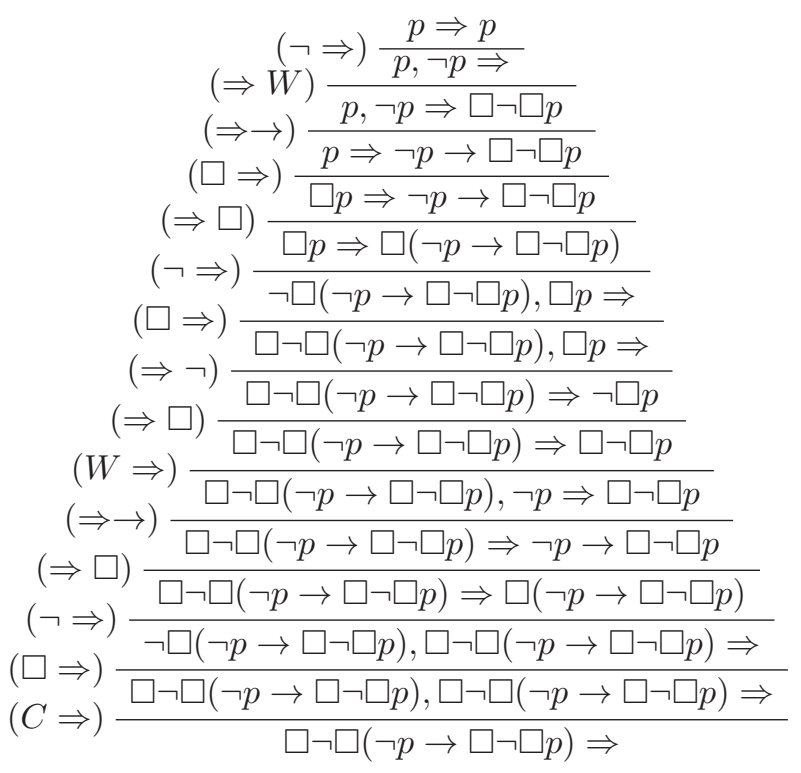


Takano [33] proposed a system of Ohnishi and Matsumoto with analytic form of cut, where cut-formula must be a subformula of other formulas in both premises. He proved constructively that every proof in Ohnishi and Matsumoto system may be transformed into a proof with only analytic cut applications. In consequence we obtain subformula-property of the system. Notice that a proof of B in Ohnishi and Matsumoto system satisfies this restriction since cut is analytic there.

Fitting on the ground of tableau calculus provided even simpler solution which may be transferred to SC. In [9] he introduced a destructive tableau system for S5 with extra rule for addition of $\square$ to formulas on the branch. It is a consequence of his completeness proof that it is sufficient to apply such a rule only once, at the very beginning. This observation is then exploited in [10] for two variants of this system differing in the definition of modalised formula. In both cases there is no extra rule for addition of $\square$ but only a requirement that when building a tableau for $\varphi$ we start with $\square \varphi$ (assumed to be false). Fitting's solution may be easily simulated in SC. One may use the following contraction-absorbing versions of Ohnishi and Matsumoto rules:

$$
(\square \Rightarrow) \frac{\varphi, \square \varphi, \Gamma \Rightarrow \Delta}{\square \varphi, \Gamma \Rightarrow \Delta} \quad(\Rightarrow \square) \frac{\square \Gamma \Rightarrow \square \Delta, \square \varphi, \varphi}{\square \Gamma \Rightarrow \square \Delta, \square \varphi}
$$

or just use Ohnishi and Matsumoto system with contraction applied before application of modal rules only when necessary. For such a system we can prove the following weak adequacy result:

THEOREM 4. $\vdash \varphi$ iff $\vdash \Rightarrow \square$

A different solution is in Bräuner [5] who provided a cut-free SC with simple modal rules on the basis of translation from $\mathrm{SC}$ for monadic firstorder classical logic. His modal rules are the following:

$$
(\square \Rightarrow) \frac{\varphi, \Gamma \Rightarrow \Delta}{\square \varphi, \Gamma \Rightarrow \Delta} \quad(\Rightarrow \square) \frac{\Gamma \Rightarrow \Delta, \varphi}{\Gamma \Rightarrow \Delta, \square \varphi}
$$

Clearly, his $(\Rightarrow \square)$ cannot be applied with no side conditions. Following Prawitz' [27] natural deduction system he stated them in terms of dependancy of occurrences of formulas in a proof. $(\Rightarrow \square)$ may be correctly applied if no occurrences of formulas in $\Gamma, \Delta$ depend on the occurrence of $\varphi$ in this proof. We direct a reader to Bräuner [5] for explication of the notion of dependency in his system. In contrast to other proposals here we 
obtain a direct constructive proof of cut elimination. On the other hand, such a system does not allow for defining a proof-search yielding a decision procedure since the control of dependency is possible rather for ready proof-trees.

It seems that the ideal system would allow both for constructive proof of cut elimination and for defining a suitable terminating proof-procedure which yields decidability. However, so far it appears possible only for nonstandard sequent calculi like labelled calculi (see e.g. Negri [20]) or hypersequent calculi for $\mathbf{S 5}$ which offer substantially enriched formal apparatus. In fact, recently Lellman and Patinson [18] provided impossibility results which imply that it is not possible to obtain a standard SC for S5 that admits standard techniques of proving cut elimination. It seems that analytic proof-search procedures may be the only possible solution for cut elimination problem in this framework.

\section{References}

[1] A. Avron, A Constructive Analysis of RM, Journal of Symbolic Logic 52 (1987), pp. 939-951.

[2] A. Avron, The method of hypersequents in the proof theory of propositional non-classical logic, [in:] W. Hodges, M. Hyland, C. Steinhorn, and J. Strauss, editors, Logic: from Foundations to Applications, Oxford University Press, Oxford (1996), pp. 1-32.

[3] K. Bednarska and A. Indrzejczak, Hypersequent calculi for S5 - the methods of cut elimination, Logic and Logical Philosophy 24/3 (2015), pp. 277-311.

[4] N. D. Belnap, Display Logic, Journal of Philosophical Logic 11(1982), pp. $375-417$.

[5] T. Braüner, A cut-free Gentzen formulation of the modal logic S5, Logic Journal of the IGPL 8 (2000), pp. 629-643.

[6] T. Brünnler, A cut-free Gentzen formulation of the modal logic S5, Logic Journal of the IGPL 8 (2000), pp. 629-643.

[7] R. Carnap, Modalities nad quantification, Journal of Symbolic Logic 11 (1946), pp. 33-64.

[8] L. Farinas del Cerro and A. Herzig, Modal Deduction with Applications in Epistemic and Temporal Logics, [in:] D. Gabbay et all. (eds.) Handbook of Logic in Artificial Intelligence and Logic Programming, vol. IV, Clarendon Press, Oxford 1995, pp. 499-594. 
[9] M. Fitting, Proof Methods for Modal and Intuitionistic Logics, Reidel, Dordrecht 1983.

[10] M. Fitting, Simple propositional S5 tableau system, Annals of Pure and Applied Logic 96 (1999), pp. 101-115.

[11] R. Goré, Tableau Methods for Modal and Temporal Logics, [in:] M. D'Agostino et al. (eds.), Handbook of Tableau Methods, Kluwer Academic Publishers, Dordrecht 1999, pp. 297-396.

[12] G. E. Hughes, M. J. Cresswell, A New Introduction to Modal Logic, Routledge, London 1996.

[13] A. Indrzejczak, Cut-free double sequent calculus for S5, Logic Journal of the IGPL 6 (1998), pp. 505-516.

[14] S. Kanger, Provability in Logic, Almqvist \& Wiksell, Stockholm 1957.

[15] S. Kripke, Semantical Analysis of Modal Logic I, Zeitschrift für Mathematische Logik und Grundlegen der Mathematik 9 (1963), pp. 67-96.

[16] H. Kurokawa, Hypersequent Calculi for Modal Logics Extending S4, [in:] New Frontiers in Artificial Intelligence, Springer 2014, pp. 51-68.

[17] O. Lahav, From Frame Properties to Hypersequent Rules in Modal Logics, [in:] Proceedings of LICS, Springer 2013, pp. 408-417.

[18] B. Lellmann, D. Pattinson, Correspondence between Modal Jilbert Axioms and Sequent Rules with an Application to S5, [in:] D. Galmiche, D. LarcheyWendling (eds.), TABLEAUX 2013. LNCS, vol. 8132, Springer 2013, pp. 219-233.

[19] G. Mints, Cut-free calculi of the S5 type, Studies in Constructive Mathematics and Mathematical Logic II (1970), pp. 79-82.

[20] S. Negri, Proof Analysis in Modal Logic, Journal of Philosophical Logic 34 (2005), pp. 507-544.

[21] M. Ohnishi, K. Matsumoto, Gentzen Method in Modal Calculi I, Osaka Mathematical Journal 9 (1957), pp. 113-130.

[22] M. Ohnishi, K. Matsumoto, Gentzen Method in Modal Calculi II, Osaka Mathematical Journal 11 (1959), pp. 115-120.

[23] F. Poggiolesi, A Cut-free Simple Sequent Calculus for Modal Logic S5, Review of Symbolic Logic 1 (2008), pp. 3-15.

[24] F. Poggiolesi, Reflecting the semantic features of S5 at the syntactic level, [in:] New Essays in Logic and Philosophy of Science, M. D'Agostino, G. Giorell, F. Laudisa, T. Pievani, C. Singaglia (eds.), London College Publications, 2010, pp. 13-25.

[25] F. Poggiolesi, Gentzen Calculi for Modal Propositional Logic, Springer 2011. 
[26] G. Pottinger, Uniform cut-free formulations of T, S4 and S5 (abstract), Journal of Symbolic Logic 48 (1983), p. 900.

[27] D. Prawitz, Natural Deduction, Almqvist and Wiksell, Stockholm 1965.

[28] G. Restall, Proofnets for S5: sequents and circuits for modal logic, [in:] Lecture Notes in Logic 28 (2007), pp. 151-172.

[29] M. Sato, A Study of Kripke-type Models for Some Modal Logics by Gentzen's Sequential Method, Publications of the Research Institute for Mathematical Sciences, Kyoto University 1977, 13, pp. 381-468.

[30] T. Skura, Refutation methods in propositional modal logics, Semper 2013.

[31] P. Stouppa, The design of modal proof theories: the case of S5, Master Thesis, Dresden 2004.

[32] P. Stouppa, A deep inference system for the modal logic S5, Studia Logica 85 (2007), pp. 199-214.

[33] M. Takano, Subformula Property as a substitute for Cut-Elimination in Modal Propositional Logics, Mathematica Japonica 37/6 (1992), pp. 1129-1145.

[34] M. Wajsberg, Ein erweiteter Klassenkalkül, Monatshefte für Mathematik unf Physik 40 (1933), pp. 113-126.

[35] H. Wansing, Displaying Modal Logics, Kluwer Academic Publishers, Dordrecht 1999.

[36] J. J. Zeman, Modal Logic, Oxford University Press, Oxford 1973.

Department of Logic

University of Eódź

Lindleya $3 / 5$

90-131 Łódź

Poland

e-mail: indrzej@filozof.uni.lodz.pl 